\title{
Kikuchi-Fujimoto Disease with Hemophagocytic Syndrome and Pleural Effusion in an Elder Man: A Case Report and Literature Review
}

\author{
Yanfang Zhang, Jianchuan Deng \\ Department of Hematology, The Second Affiliated Hospital of Chongqing Medical University, Chongqing, China \\ Email: zhangyanfang6868@163.com,dengjccq@hospital.cqmu.edu.cn
}

How to cite this paper: Zhang, Y.F. and Deng, J.C. (2020) Kikuchi-Fujimoto Disease with Hemophagocytic Syndrome and Pleural Effusion in an Elder Man: A Case Report and Literature Review. Case Reports in Clinical Medicine, 9, 311-318. https://doi.org/10.4236/crcm.2020.910044

Received: August 25, 2020

Accepted: October 16, 2020

Published: October 19, 2020

Copyright (อ 2020 by author(s) and Scientific Research Publishing Inc. This work is licensed under the Creative Commons Attribution International License (CC BY 4.0).

http://creativecommons.org/licenses/by/4.0/

\section{(c) (i) Open Access}

\begin{abstract}
Background: Kikuchi-Fujimoto disease (KFD), also named Histiocytic necrotizing lymphadenitis (HNL), is a rare and self-limited necrotizing lymphadenitis, most commonly affects young Asian female. KFD is characterized by lymphadenopathy, cytopenia, fever, rashes, and weight loss, however, hemophagocytic syndrome and pleural effusion are rarely reported. Case Presentation: We present the case of a 61-year-old man with clinical features including fever, lymphadenopathy, purpura, arthralgia, pleural effusion and pulmonary infection. He had cervical lymphnodebiopsy and the pathology is consistent with KFD, laboratory tests and clinical manifestations were consistent with hemophagocytic syndrome (HLH). After initial treatment including corticosteroid and anti-infection drugs, the patient showed improvement but soon deteriorated. Conclusion: KFD is a self-limited disease with spontaneous resolution. However, the cases of KFD with HLH may have a fatal course. Early appropriate intensive immunosuppressive therapy and strong anti-infection therapy could be beneficial for the treatment outcome.
\end{abstract}

\section{Keywords}

Kikuchi-Fujimoto Disease, Hemophagocytic Syndrome, Pleural Effusion

\section{Introduction}

Kikuchi-Fujimoto disease (KFD), also named Histiocytic necrotizing lymphadenitis (HNL), is a rare and self-limited necrotizing lymphadenitis which was initially described by Japanese pathologists Kikuchi and Fujimoto in 1972. It is always characterized by cervical lymphadenopathy, cytopenia, fever and weight 
loss, and about $16.6 \%$ - $40 \%$ have varied rashes [1] [2]. In general, KFD is more common among women in particular young Asian females but it has a worldwide distribution [3]. The KFD with the hemophagocytic syndrome and pleural effusion is rarely reported. Here, we report an elder Asian man who experienced unexplained fever, swelling of the generalized lymph node, bilateral pleural effusion, anaphylacto purpura and hemophagocytic syndrome, and was ultimately diagnosed KFD based on the lymph node biopsy.

\section{Case Report}

A 61-year-old man was admitted to our hospital due to recurrent fever and arthralgia for more than one month. Before admission, the patient began to experience general arthralgia and fever, the temperature was between $38.0^{\circ} \mathrm{C}$ and $39^{\circ} \mathrm{C}$, without cough, expectoration, dizziness, headache, abdominal pain, diarrhea et al. Then he went to the Chongqing fifth people's hospital, laboratory findings were as follows: hemoglobin $88 \mathrm{~g} / \mathrm{l}$, leukocyte count $4.9 \times 10^{12} / \mathrm{L}$, neutrophils $77.1 \%$, platelet count $91 \times 10^{9} / \mathrm{L}$, computed tomography $(\mathrm{CT})$ of the chest revealed double emphysema, fibrous foci in the middle lobe of the right lung, patchy density increasing shadow in the lower lobe of the left lung, considered to be infected, a small amount of effusion in the left thoracic cavity. He received anti -bacterial, antipyretic treatment, however, he still had a fever and muscular soreness. Whereafter he went to our hospital and was hospitalized in the geriatrics department on April 1st, 2020.

In our hospital, laboratory examinations revealed as following: red blood cell count $2.19 \times 10^{12} / \mathrm{L}$, hemoglobin $74 \mathrm{~g} / \mathrm{L}$, white blood cell count $14.70 \times 10^{9} / \mathrm{L}$, neutrophils $85.9 \%$, platelets $126 \times 10^{9} / \mathrm{L}$, C-reactive protein: $117.22 \mathrm{mg} / \mathrm{l}$, erythrocyte sedimentation rate $110 \mathrm{~mm} / \mathrm{h}$; procalcitonin $0.1 \mathrm{ng} / \mathrm{ml}$; immune globulin IgA $5.16 \mathrm{~g} / \mathrm{L}, \operatorname{IgM} 0.44 \mathrm{~g} / \mathrm{L}$, rheumatoid factor (RF) $23.22 \mathrm{IU} / \mathrm{ml}$, ferritin 936 $\mathrm{ng} / \mathrm{ml}$, serum antinuclear antibody (ANA) positive (1:100), serum lactic dehydrogenase (LDH) $361 \mathrm{IU} / \mathrm{L}$, anti-nuclear antibody and anti-neutrophil cytoplasmic antibodies were normal. The T-SPOT, purified protein derivative tests for tuberculosis and serologic tests for human immunodeficiency virus (HIV), hepatitis B surface antigen and hepatitis C antibody, Epstein-Barr virus (EBV), cytomegalovirus (CMV) were all negative. Bone marrow cytological analysis revealed bone marrow hyperplasia. Computed tomography (CT) of the chest and abdomen revealed bilateral pleural effusion, which is more severe on the left side. No lymph node and enlargement nor hepatosplenomegaly were found (see Figure 1). After admission, the patient received anti-bacteria with carbapenems antibiotics treatment. After 3 weeks of treatment, the patient still experienced recurrent fever. He was suspected of hemophagocytic syndrome, so transferred to our department. In our department, his laboratory examinations as follows: cytokine detection elevated interleukin-2 receptor (IL-2R) $4051.00 \mathrm{U} / \mathrm{ml}$, ferritin higher than $1500 \mathrm{ng} / \mathrm{ml}$, haemophilus influenza and herpes simplex were positive. Reviewed the bone marrow found a few atypical lymphocytes, plasma cells, 


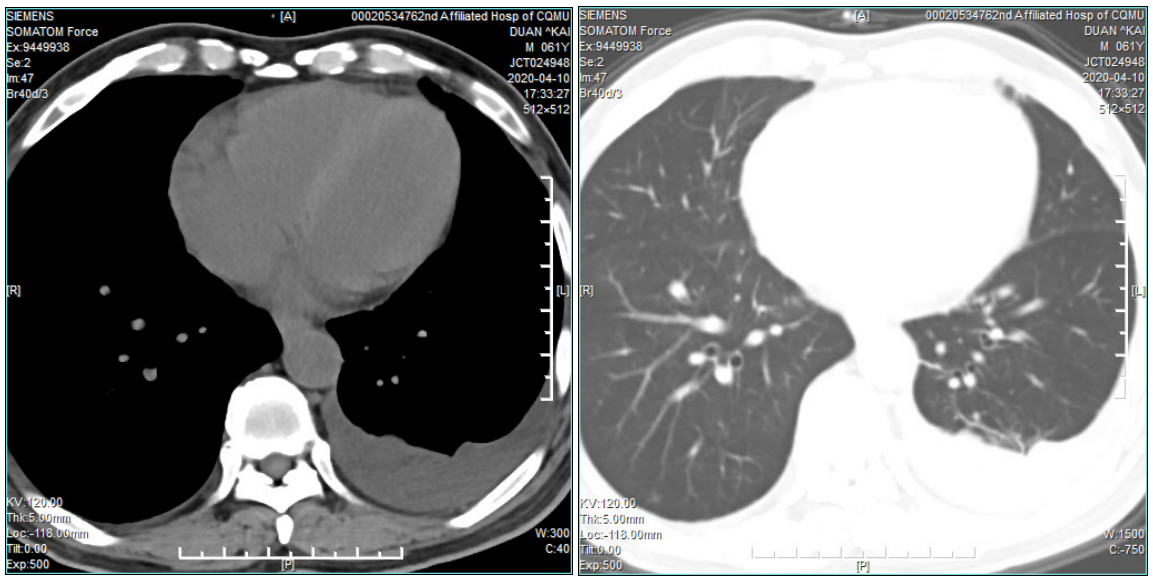

Figure 1. On admission bilateral pleural effusion, more severe on the left side, no lymph node enlargement was found.

reticular cells, and hemophagocytosis. Reexamination of the computed tomography (CT) of the chest revealed bilateral pleural effusion was slightly increased, right lung inflammation, bilateral axillary, hilar and mediastinal lymph nodes were newly enlarged (see Figure 2). The patient was diagnosed with the hemophagocytic syndrome, according to the HLH 2004 clinical trial diagnostic guidelines [4], and dexamethasone was used to control hemophagocytosis combined with antibacterial and an empirical antifungal treatment. Soon afterwards, the patient gradually appeared bilateral lower limb purpura, allergic purpura was considered. Examination of pleural effusion: the nuclear cells: $1592.0 \times 10^{6} / \mathrm{L}$, neutrophils: $25 \%$, lymphocytes: $60 \%$, mesothelial cells: $14 \%$, glucose 10.24 $\mathrm{mmol} / \mathrm{L}$, total protein $31.9 \mathrm{~g} / \mathrm{L}$, albumin $16.8 \mathrm{~g} / \mathrm{L}$, globulin $15.1 \mathrm{~g} / \mathrm{L}, \mathrm{LDH} 125$ U/L, ADA $5.8 \mathrm{U} / \mathrm{L}$. Pleural effusion mycobacterium tuberculosis smear was negative. The flow cytometry of pleural effusion detected CD4+CD8+ cells accounted for $5.02 \%$, with an increased proportion. Plasma cells accounted for $15.97 \%$ of the total number of cells which were polyclonal. A biopsy on the left cervical lymph node, no abnormalities were found in lymph node flow cytometry, pathological examination showed scattered fibrin deposition, a large amount of nuclear debris and large mononuclear cell aggregates in the necrotic area (see Figure 3), and the immunohistochemical showed CD3T cell(+) CD20B cell( $(+)$ CD21FDC(+) CD23 FDC(+) Ki6730\%(+) Des(-) LCA lymphocyte(+) S100(-) Langerin(-) CDla(-) CD5T cell(+) CD68 histocyte(+) CD4T cell(+) CD8T cell(+) CD43T cell(+) MPO(str + ) CD19B cell(+) CD10(+) BCL-2 T cell(+) BCL-6(+) CK(-) EMA(-) Vim mesenchyme(+) TDT(-), the pathological diagnosis was histiocytic necrotizing lymphadenitis. As for the treatment of the KFD, along with hemophagocytic syndrome and pulmonary infection, we combined dexamethasone with anti-infection treatment. Because the dexamethasone therapy was already started on the day he was diagnosed with hemophagocytic syndrome, he had rapid improvement of all clinical symptoms, body temperature return to normal, cervical and axillary swelling and tenderness began to decrease, purpura of lower limbs faded rapidly, the pleural effusion and pulmonary 


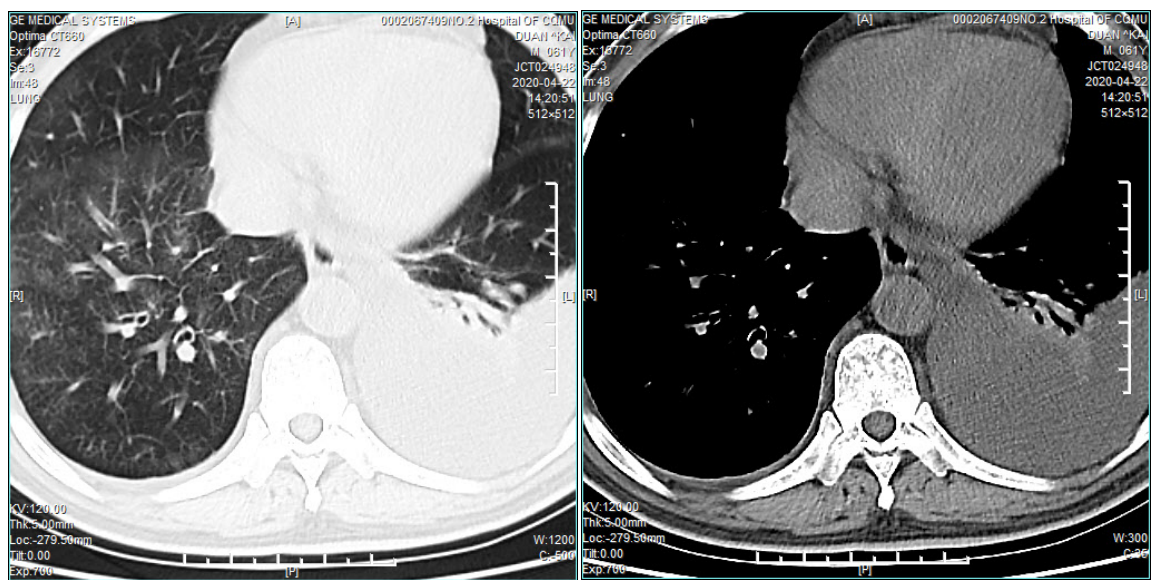

Figure 2. Reexamination the computed tomography (CT) of the chest revealed bilateral pleural effusion was slightly increased, right lung inflammation, bilateral axillary, hilar and mediastinal lymph nodes were newly enlarged.

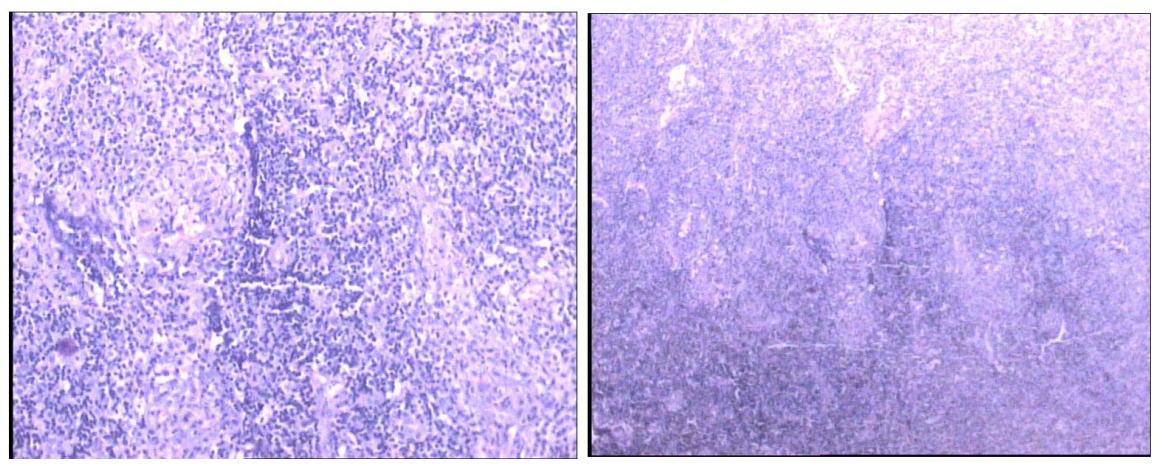

Figure 3. Light microscopy of the lymph node biopsy, hematoxylin and eosin $\times 400$. Necrosis, nuclear debris and plasma cells are present, scattered fibrin deposition, a large amount of nuclear debris and large mononuclear cell aggregates in the necrotic area.

inflammation disappeared (see Figure 4). The patient was discharged from the hospital after 1-month of treatment. However, a few days later, he was readmitted to the hospital due to severe pneumonia (see Figure 5), eventually transferred to the intensive care unit (ICU) due to acute respiratory failure. So far, he had already got out from the ICU, but still underwent almost four months anti-infection in the hospital.

\section{Discussion}

Kikuchi-Fujimoto disease (KFD) is a rare, benign disease, presenting with lymphadenopathy, frequently associated with a series of systemic manifestations such as fever, cutaneous eruptions, cytopenia and so on [5]. The aetiology of KFD remains unclear, and may associate with viral infection or autoimmune diseases [6] [7] [8] [9]. The pathogenesis of KFD, may involve the activation of CD8+ T cell, leads to T cell proliferation and dying via the Fas and perforin pathways; then, macrophages phagocytic apoptotic cells, and resulting in typical necrotizing lymphadenitis [10]. However, in a meta-analysis using 41 studies, 


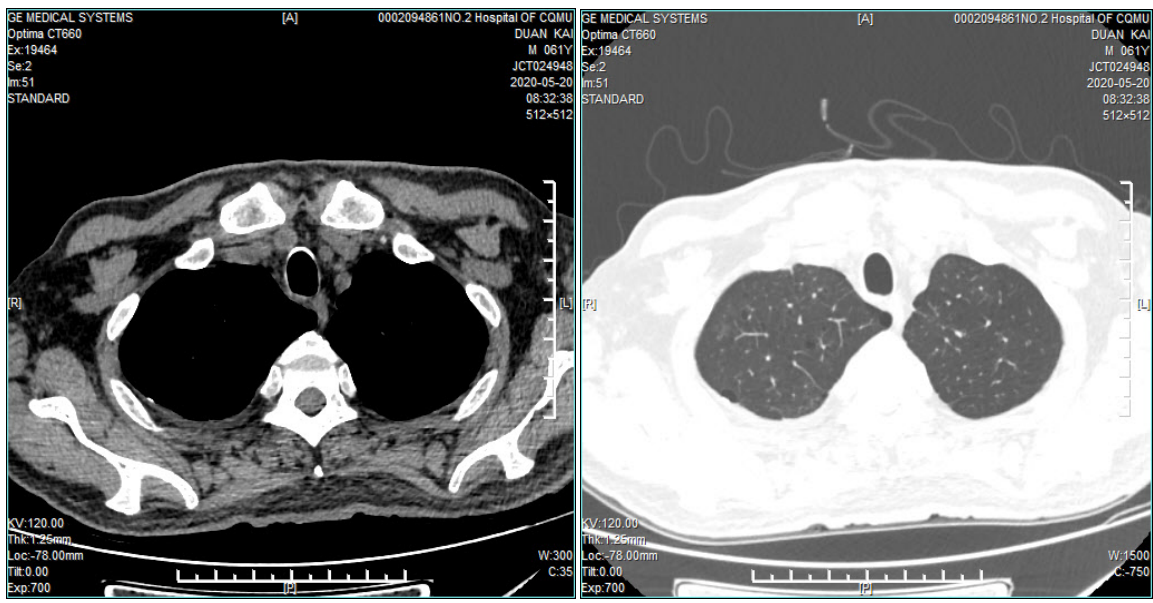

Figure 4. 1 month after treatment, the patient got a rapid improvement, pulmonary inflammation and pleural effusion were significantly reduced, lymph node reduction.

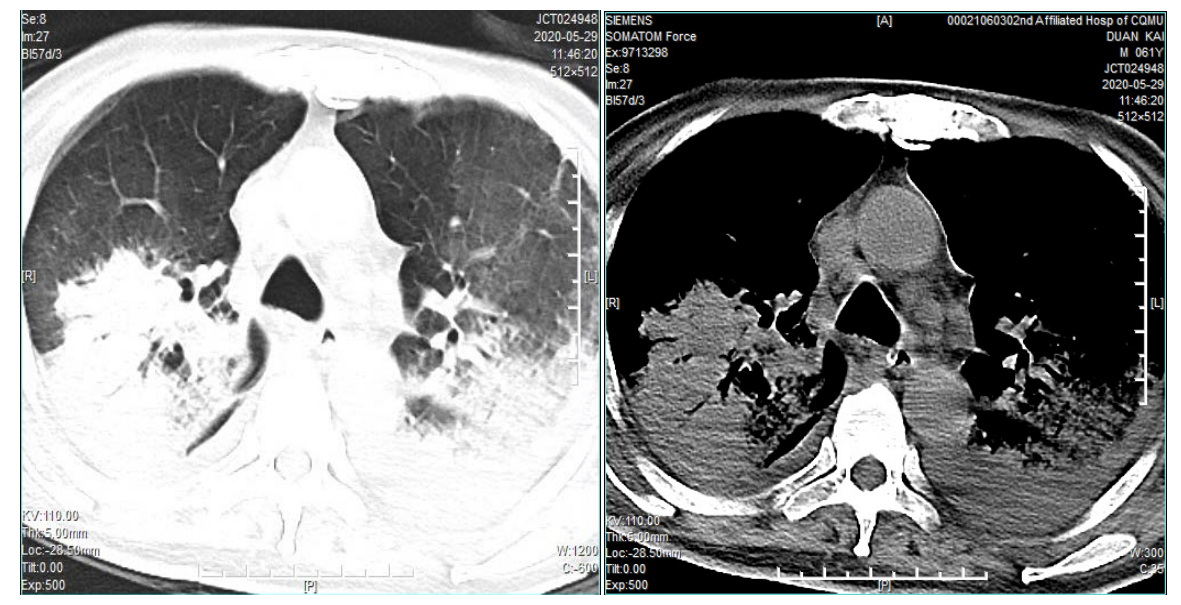

Figure 5. A few days after discharge, he was readmitted to hospital due to severe pneumonia, chest CT showed a significant increase in pulmonary infection and pleural effusion.

none of the most pathogenic virus including Epstein-Barr virus (EBV), human herpesvirus, human herpesvirus 8, HIV, parvovirus B19, cytomegalovirus, hepatitis B virus (HBV), and human T-lymphotropic virus 1 (HTLV-1), were related to KFD [11]. Other reports suggested immune disorder maybe an important pathogenesis [6] [12] [13] [14] [15]. Since Most cases were showed KFD can occur before, after or concurrently with SLE, particular in these share same characteristics: child-bearing period female, fever, lymphadenopathy, cytopenia, arthralgia and skin lesions [14]-[19]. Some scholars even hold that KFD is a unique manifestation of SLE [20]. In a 2007 case report which included 244 cases of KFD, showed that the SLE occurred together in $13 \%$ of cases, $9.8 \%$ of non-infectious inflammatory diseases and 7\% of viral infections [21]. Our patient showed a specific pathogen pulmonary infection along with the KFD, and no immunological indicators positive, so the infection may be considered the pathogenic factor. 
As for the clinical manifestation, fever, cytopenia, lymphadenopathies, cutaneous eruptions are the most relevant manifestations of KFD. However KFD with pulmonary involvement and pleural effusion has rarely been reported, and most such cases with concomitant of autoimmune disease or pulmonary infection, only few pleural effusion isolated with KFD [6] [21] [22]. For our patient, the pleural effusion along with pulmonary infection, the analysis of the pleural effusion showed lymphocyte account for a majority, and the cytometry detected an increased proportion of $\mathrm{CD} 4+\mathrm{CD} 8+$ cells, however anti-infection treatment did not reduce the effusion, but significantly decreased with steroid. So we regarded the likelihood that KFD had pleural effusion.

Hemophagocytic syndrome (HPS) is also an unusual disorder characterized by fever, cytopenia, hepatosplenomegaly, inflammatory cytokine storm caused by natural killer (NK) cell activity decreased, mononuclear macrophage activation, and hemophagocytosis in the reticuloendothelial system [23]. HPS was rarely associated with KFD or SLE. Frikha F et al., presented a report in 2008, was the first case of fatal KFD simultaneously occurred with SLE complicated with HLH and severe infection [10]. But the HPS was associated with SLE, the proposed pathogenesis is immune complexes activate the CD8+ T cell, result in oversecretion of various cytokines by uncontrolled $\mathrm{T}$ cell activation, then apoptotic cells are engulfed by macrophages [24]. The outcome of HPS is usually aggressive and fatal in many cases, as the same with this patient. Although for our patient, HPS occurring as the initial presentation of KFD, and no immunological indicators positive, he also had an unfavorable prognosis.

\section{Conclusions}

For our patient, he had no cause of autoimmune disease, but he had pulmonary infection which may play a key role cause for KFD and secondary HLH. The pleural effusion may triggered by KFD. Hemophagocytic syndrome, occurring as the initial presentation of KFD and worsened during the clinical course, may originate from concurrent severe pulmonary infection. KFD along with HPS contributed to the patient's deterioration.

KFD is a self-limited disease with spontaneous resolution within 4 months. However, the cases of KFD with severe pulmonary infection and secondary HLH may have a fatal course, like the presented patient. Early appropriate intensive immunosuppressive therapy and strong anti-infection therapy could be beneficial for the treatment outcome.

\section{Acknowledgements}

Thank Dr YanShen for providing purely technical help and writing assistance.

\section{Consent}

Written informed consent was obtained from the patient for publication of this case report and any accompanying images. 


\section{Conflicts of Interest}

The authors declare no conflicts of interest regarding the publication of this paper.

\section{References}

[1] Morishima, T., Mizutani, Y., Takahashi, T., Miyazaki, T. and Seishima, M. (2018) Histiocytic Necrotizing Lymphadenitis (Kikuchi's Disease) with Vasculitis. The Journal of Dermatology, 45, e98-e99. https://doi.org/10.1111/1346-8138.14143

[2] Kuo, T.T. (1990) Cutaneous Manifestation of Kikuchi's Histiocytic Necrotizing Lymphadenitis. The American Journal of Surgical Pathology, 14, 872-876. https://doi.org/10.1097/00000478-199009000-00009

[3] Onciu, M. and Medeiros, L.J. (2003) Kikuchi-Fujimoto Lymphadenitis. Advances in Anatomic Pathology, 10, 204-211. https://doi.org/10.1097/00125480-200307000-00003

[4] Zhang, J.R., Liang, X.L., Jin, R. and Lu, G. (2013) HLH-2004 Protocol: Diagnostic and Therapeutic Guidelines for Childhood Hemophagocytic Lymphohistiocytosis. Chinese Journal of Contemporary Pediatrics, 15, 686-688.

[5] Lamzaf, L., Harmouche, H., Maamar, M., Adnaoui, M., Aouni, M. and Tazi Mezalek, Z. (2014) Kikuchi-Fujimoto Disease: Report of 4 Cases and Review of the Literature. European Annals of Oto-rhino-laryngology, Head and Neck Diseases, 131, 329-332. https://doi.org/10.1016/j.anorl.2013.01.007

[6] Chen, H.C., Lai, J.H., Huang, G.S., Gao, H.W., Chen, C.H., Kuo, S.Y. and Chang, D.M. (2005) Systemic Lupus Erythematosus with Simultaneous Onset of Kikuchi-Fujimoto's Disease Complicated with Antiphospholipid Antibody Syndrome: A Case Report and Review of the Literature. Rheumatology International, 25, 303-306. https://doi.org/10.1007/s00296-004-0507-4

[7] Garcia-Zamalloa, A., Taboada-Gomez, J., Bernardo-Galán, P., Magdalena, F.M., Zaldumbide-Dueñas, L. and Ugarte-Maiztegui, M. (2010) Bilateral Pleural Effusion and Interstitial Lung Disease as Unusual Manifestations of Kikuchi-Fujimoto Disease: Case Report and Literature Review. BMC Pulmonary Medicine, 10, 54. https://doi.org/10.1186/1471-2466-10-54

[8] Hudnall, S.D., Chen, T., Amr, S., Young, K.H. and Henry, K. (2008) Detection of human Herpesvirus DNA in Kikuchi-Fujimoto Disease and Reactive Lymphoid Hyperplasia. International Journal of Clinical and Experimental Pathology, 1, 362-368.

[9] Chong, Y., Lee, J.Y., Thakur, N., Kang, C.S. and Lee, E.J. (2020) Strong Association of Torque Teno Virus/Torque Teno-Like Minivirus to Kikuchi-Fujimoto Lymphadenitis (Histiocytic Necrotizing Lymphadenitis) on Quantitative Analysis. Clinical Rheumatology, 39, 925-931. https://doi.org/10.1007/s10067-019-04851-4

[10] Kampitak, T. (2008) Fatal Kikuchi-Fujimoto Disease Associated with SLE and Hemophagocytic Syndrome: A Case Report. Clinical Rheumatology, 27, 1073-1075. https://doi.org/10.1007/s10067-008-0902-4

[11] Chong, Y. and Kang, C.S. (2014) Causative Agents of Kikuchi-Fujimoto Disease (Histiocytic Necrotizing Lymphadenitis): A Meta-Analysis. International Journal of Pediatric Otorhinolaryngology, 78, 1890-1897.

https://doi.org/10.1016/j.ijporl.2014.08.019

[12] Papaioannou, G., Speletas, M., Kaloutsi, V. and Pavlitou-Tsiontsi, A. (2002) Histiocytic Necrotizing Lymphadenitis (Kikuchi-Fujimoto Disease) Associated with An- 
tiphospholipid Syndrome: Case Report and Literature Review. Annals of Hematology, 81, 732-735. https://doi.org/10.1007/s00277-002-0562-4

[13] Kim, S.K., Kang, M.S., Yoon, B.Y., Kim, D.Y., Cho, S.K., Bae, S.C. and Her, M.Y. (2011) Histiocytic Necrotizing Lymphadenitis in the Context of Systemic Lupus Erythematosus (SLE): Is Histiocytic Necrotizing Lymphadenitis in SLE Associated with Skin Lesions? Lupus, 20, 809-819. https://doi.org/10.1177/0961203310397684

[14] Ruaro, B., Sulli, A., Alessandri, E., Fraternali-Orcioni, G. and Cutolo, M. (2014) Kikuchi-Fujimoto's Disease Associated with Systemic Lupus Erythematous: Difficult Case Report and Literature Review. Lupus, 23, 939-944. https://doi.org/10.1177/0961203314530794

[15] Vithoosan, S., Karunarathna, T., Shanjeeban, P., Piranavan, P., Matthias, T., Gamlaksha, D., Liyadipita, A. and Kulatunga, A. (2019) Kikuchi-Fujimoto Disease Associated with Systemic Lupus Erythematosus Complicated with Hemophagocytic Lymphohistiocytosis: A Case Report. Journal of Medical Case Reports, 13, 173. https://doi.org/10.1186/s13256-019-2100-1

[16] Baenas, D.F., Diehl, F.A., Haye Salinas, M.J., Riva, V., Diller, A. and Lemos, P.A. (2016) Kikuchi-Fujimoto Disease and Systemic Lupus Erythematosus. International Medical Case Reports Journal, 9, 163-167. https://doi.org/10.2147/IMCRJ.S106396

[17] Găman, M., Vlădăreanu, A.M., Dobrea, C., Onisâi, M., Marinescu, C., Voican, I., Vasile, D., Bumbea, H. and Cîşleanu, D. (2018) A Challenging Case of Kikuchi-Fujimoto Disease Associated with Systemic Lupus Erythematosus and Review of the Literature. Case Reports in Hematology, 2018, Article ID: 1791627. https://doi.org/10.1155/2018/1791627

[18] Pace-Asciak, P., Black, M.A., Michel, R.P. and Kost, K. (2008) Case Series: Raising Awareness about Kikuchi-Fujimoto Disease among Otolaryngologists: Is It Linked to Systemic Lupus Erythematosus? Journal of Otolaryngology_Head \& Neck Surgery, 37, 782-787.

[19] Frikha, F., Marzouk, S., Frigui, M., Jallouli, M., Kechaou, M., Kaddour, N., Boudawara, T., Jlidi, R. and Bahloul, Z. (2008) Kikuchi-Fujimoto's Disease and Connective Tissue Disease: A Report of Three Cases. La Revue de Médecine Interne, 29, 129-134. https://doi.org/10.1016/j.revmed.2007.07.012

[20] Yu, H.L., Lee, S.S., Tsai, H.C., Huang, C.K., Chen, Y.S., Lin, H.H., Wann, S.R., Liu, Y.C. and Tseng, H.H. (2005) Clinical Manifestations of Kikuchi's Disease in Southern Taiwan. Journal of Microbiology, Immunology and Infection, 38, 35-40.

[21] Kucukardali, Y., Solmazgul, E., Kunter, E., Oncul, O., Yildirim, S. and Kaplan, M. (2007) Kikuchi-Fujimoto Disease: Analysis of 244 Cases. Clinical Rheumatology, 26, 50-54. https://doi.org/10.1007/s10067-006-0230-5

[22] Sharma, O.P. (2001) Unusual Systemic Disorders Associated with Interstitial Lung Disease. Current Opinion in Pulmonary Medicine, 7, 291-294. https://doi.org/10.1097/00063198-200109000-00007

[23] Zhuo, W.B., Gao, Y., Yang, C.Y., Xu, Y., Zhang, Y.T., Zhu, D.M. and Ping, B.H. (2018) Clinical Characteristics of Hemophagocytic Syndrome: Analysis of 46 Cases. Journal of Southern Medical University, 38, 769. https://doi.org/10.1182/blood-2018-99-114383

[24] Romanou, V., Hatzinikolaou, P., Mavragani, K.I., Meletis, J. and Vaiopoulos, G. (2006) Lupus Erythematosus Complicated by Hemophagocytic Syndrome. Journal of Clinical Rheumatology, 12, 301-303. https://doi.org/10.1097/01.rhu.0000249897.14441.c6 